\title{
Evaluación del Consumo de Drogas en Adolescentes con y sin TDAH. Nuevas Evidencias y Recomendaciones
}

\author{
Evaluation of Drug Use in Adolescents with and without ADHD. New Evidences and \\ Recommendations
}

\author{
Manuel Isorna Folgar ${ }^{1}$, David Liñares Mariñas ${ }^{2}$, Sandra Golpe Ferreiro ${ }^{3}$, \\ Patricia Gómez Salgado ${ }^{4}$ y Antonio Rial Boubeta ${ }^{5}$
}

\begin{abstract}
Resumen
El consumo intensivo de alcohol, el descenso de las edades de inicio, el repunte del consumo de cannabis, o las elevadas tasas de consumo en mujeres, son algunos de los nuevos hallazgos en el estudio de las adicciones. Paralelamente, diversas investigaciones constatan un mayor consumo de drogas en los adolescentes diagnosticados con TDAH. Sin embargo, son escasos los estudios que analizan los consumos de drogas y los consumos de riesgo en adolescentes con TDAH en su propio contexto socioeducativo. Muestra: 3339 adolescentes entre 12 y 18 años (222 con TDAH), pertenecientes a 12 centros escolares. Resultados: las chicas con TDAH muestran tasas significativamente mayores de consumo de alcohol ( $70 \%$ vs $50.1 \%$ ), tabaco $(54.3 \%$ vs $28 \%)$ y cannabis $(28.6 \%$ vs. $13.9 \%)$ comparadas con sus compañeras sin TDAH, obteniéndose también diferencias en los consumos de riesgo y en las edades de inicio de las diferentes drogas.
\end{abstract}

Palabras clave: adolescentes, TDAH, drogas, diferencias, género, prevención

\begin{abstract}
Intensive alcohol consumption, the decrease in the starting ages, the rise in cannabis use, or the high rates of consumption in women, are some of the new findings in the study of addictions. At the same time, different studies show a higher consumption of substances in adolescents diagnosed with ADHD. However, there are still few studies that have analysed the consumption habits and the possible consumption risk in adolescents with ADHD in their socio-educational context. The sample used in the present work consists of 3339 adolescents between 12 and 18 years old ( 222 with ADHD) belonging to 12 schools. It was found that girls with ADHD show significantly higher rates of alcohol consumption (70\% vs. $50.1 \%)$, tobacco (54.3\% vs. $28 \%$ ) and cannabis (28.6\% vs. $13.9 \%$ ), also obtaining alarming differences in the consumption risk and in the onset ages for different substances.
\end{abstract}

Keywords: adolescents, ADHD, drugs, gender differences, prevention

Agradecimientos: Los autores de este trabajo quieren agradecer la financiación recibida a través de la Delegación del Gobierno para el Plan Nacional sobre Drogas (Ref. 2018/008) para la realización de este estudio.

\footnotetext{
${ }^{1}$ Universidad de Vigo. Facultad Ciencias Educación y Trabajo Social. Campus de Ourense. As Lagoas. CP. 32004. Ourense. Telf. 988387100. Correo: isorna.catoira@uvigo.es (Autor de correspondencia)

2 Investigador del grupo Psicom. Rúa de José María Suárez Núñez, s/n, Santiago de Compostela, A Coruña (A Coruña). Correo: davidlinares.marinas@usc.es

${ }^{3}$ Doctora en Psicología. Investigadora del grupo Psicom. Rúa de José María Suárez Núñez, s/n, Santiago de Compostela, A Coruña (A Coruña). Correo: sandra_golpe@hotmail.com

${ }^{4}$ Doctora en Psicología. Investigadora del grupo Psicom. Rúa de José María Suárez Núñez, s/n, Santiago de Compostela, A Coruña (A Coruña). Correo: patricia.gomez@usc.es

${ }^{5}$ Doctor en Psicología. Profesor titular. Grupo Psicom. Rúa de José María Suárez Núñez, s/n, Santiago de Compostela, A Coruña (A Coruña). Correo: antonio.rial.boubeta@usc.es 


\section{Introducción}

Los datos reflejados por el Observatorio Español de Drogas y las Adicciones [OEDA] (2020) ponen de manifiesto que el consumo abusivo de drogas entre los adolescentes constituye uno de los principales problemas de salud pública en España. Habida cuenta del interés que este tema sigue suscitando a diferentes niveles, la Estrategia Nacional sobre Adicciones 2017-2024 (PNSD, 2018) recoge una serie de medidas para reducir en la medida de lo posible este fenómeno. En todo caso, hoy en día se presentan una serie de hallazgos de suma relevancia en el consumo de sustancias entre los adolescentes.

Por un lado, tal y como muestra el OEDA (2020) la prevalencia del consumo de alcohol en los estudiantes de Educación Secundaria de 14-18 años ha sido de un $77.9 \%$ alguna vez en la vida, el $75.9 \%$ en el último año y el $58.5 \%$ en el último mes. En cuanto a la incidencia, en 2018, se iniciaron en el consumo de alcohol 346600 estudiantes.

En todo caso el patrón de consumo ha empeorado en peligrosidad y riesgo. Así en la literatura, son habituales términos como el consumo intensivo de alcohol [CIA] (RodríguezMartos \& Rosón, 2008) o binge drinking [BD] (Parada et al., 2011) para alertar del riesgo que suponen estas nuevas formas de consumo. En España se considera CIA al consumo de 5 o más bebidas alcohólicas en hombres y 4 o más en mujeres en una ocasión (aproximadamente en el trascurso de 2 horas). En 2018, un 32.3\% de los estudiantes ha consumido con el patrón de binge drinking en el último mes y casi la mitad de los estudiantes se ha emborrachado en el último año, el $46.5 \%$ de las chicas y el $42.3 \%$ de los chicos.

En cuanto al consumo de tabaco, el OEDA (2020) muestra que el $41.3 \%$ de los estudiantes entre 14-18 años ha fumado tabaco alguna vez en la vida, el $26.7 \%$ confirma un consumo en el último mes y el $9.8 \%$ manifiesta un consumo diario. Se estima que en 2018 se han iniciado en el consumo de tabaco unos 205600 nuevos jóvenes. No debemos olvidar que, tal y como recoge Rial, Golpe, Barreiro, Gómez y Isorna (2018), el tabaco junto con el alcohol son las sustancias que constituyen la "puerta de entrada" al consumo de otras sustancias psicoactivas tanto legales como ilegales.

Siguiendo con los datos recogidos en el OEDA (2020), el cannabis sigue siendo la droga ilegal más consumida, así en 2018 el 33\% había consumido cannabis alguna vez en la vida, el $27.5 \%$ en el último año y el $19.3 \%$ en el último mes. Entre los que consumieron en el último año, el $3.3 \%$ de los chicos y el $1.4 \%$ de las chicas han consumido 20 días o más. Iniciándose en su consumo cerca de 222200 jóvenes en 2018.

En cuanto al consumo de cocaína, en 2018, el $2.9 \%$ de la población de 14 a 18 años ha consumido cocaína (polvo y/o base) alguna vez en la vida, el $2.4 \%$ en el último año y el $0.9 \%$ en el último mes. En cuanto al consumo en el último año, el 1.9\% de los jóvenes reconoce el consumo de éxtasis, el $1.1 \%$ de anfetaminas y el $1.4 \%$ alucinógenos.

Otro dato a tener en cuenta es el descenso en la edad de inicio en el consumo drogas en los adolescentes. Numerosos trabajos han señalado que los adolescentes que comienzan a consumir a una menor edad presentan: un mayor riesgo de sufrir daños cerebrales y consecuencias a nivel neurocognitivo, la probabilidad de verse implicados en numerosas prácticas de riesgo (tales como peleas o actos de violencia), un peor rendimiento académico, prácticas sexuales de riesgo y accidentes de tráfico (Carbia, LópezCaneda, Corral, \& Cadaveira, 2018; Golpe, Barreiro, Isorna, Varela, \& Rial, 2017). Por lo tanto, no es de extrañar que el retraso de la edad de inicio siga siendo un reto ya recurrente, presentándose como el tercer objetivo general tanto en la pasada Estrategia Nacional sobre Drogas 2009-2016 (PNSD, 2009), como en la reciente Estrategia Nacional sobre Adicciones 2017-2024 (PNSD, 2017).

Desde hace más de una década, uno de los grandes objetivos y retos en la investigación en adicciones, es la inclusión de los perfiles diferenciales entre hombres y mujeres y la perspectiva de género (Observatorio Europeo de las Drogas y las Toxicomanías, 2006; Vázquez, Isorna, \& Redondo, 2020). En el Informe del Observatorio de Proyecto Hombre sobre el perfil de las personas con problemas de adicción en tratamiento (Asociación Proyecto Hombre, 2016) se ha podido constatar un incremento en el 
número de mujeres que acuden a tratamiento con problemas de adicción a drogas, pasando de un 14\% en el año 2012 a un $16 \%$ en el año 2016.

Paralelamente a las conductas adictivas, el Trastorno por Déficit de Atención e Hiperactividad [TDAH] se ha convertido en otro de los trastornos de interés a nivel científico en el ámbito infanto-juvenil. Según el Manual Diagnóstico y Estadístico de los Trastornos Mentales [DSM-5] (Asociación Americana de Psiquiatría, 2014), el TDAH es un trastorno del neurodesarrollo con bases neurobiológicas, cuyos rasgos principales son, por una parte, la dificultad para sostener la concentración (déficit de atención) en circunstancias que ofrecen baja estimulación y, por otra, la falta de inhibición o control cognitivo sobre los impulsos, frecuentemente asociadas con inquietud motora (hiperactividad-impulsividad). Estos dos conjuntos de signos pueden aparecer por separado o combinados. Además, es un trastorno muy frecuente, que afecta al $8 \%$ de los adolescentes de entre 13 y 18 años (Willcutt, 2012). En España se ha establecido que, aproximadamente, el TDAH es un trastorno que afecta entre un 5\% y $6.8 \%$ de los jóvenes (Catalá-López et al., 2012; Isorna, Rial, Felpeto, \& Mociño, 2017; Rivas, de Dios, Benac, \& Colomer, 2017). A la gran prevalencia de este trastorno, hay que sumarle las alteraciones y consecuencias negativas que suele provocar en los individuos que lo padecen y que se manifiestan en: bajo rendimiento escolar y un desempeño inferior en la mayoría de las pruebas que evalúan funciones ejecutivas (Rubiales, 2014), aislamiento provocado por rechazo de los iguales, falta de autoestima, agresividad, problemas de convivencia en el hogar, problemas asociados a conductas inapropiadas como mayor número de accidentes y el consumo de drogas (Isorna, 2015). Uno de los aspectos que mayor interés ha despertado en la comunidad científica es la alta comorbilidad en este trastorno (Quintero, Loro, Jiménez, \& Campos, 2011); las investigaciones llevadas a cabo estiman que existen trastornos comórbidos al TDAH en el 15\% y el 68\% de los casos (Bloemsma et al., 2013; González-Castro, Rodríguez, Cueli, Cabeza, \& Álvarez, 2014; Reale et al., 2017). Estudios científicos y clínicos destacan la relación del TDAH con el consumo de sustancias. Así, hay investigaciones que ponen de manifiesto que un $25.3 \%$ de adolescentes con trastorno por consumo de sustancias están diagnosticados con TDAH (van Emmerik-van Oortmerssen et al., 2012; Daigre, Terán, García-Vicent, \& Roncero, 2013). Además, los sujetos que en su infancia presentaron TDAH, inician el consumo de sustancias a una edad más temprana y evolucionan hacia un trastorno por abuso y/o dependencia más fácil y rápidamente que los que no tienen este trastorno (Pérez de los Cobos et al., 2011; Miranda, Colomer, Berenguer, Roselló, \& Roselló, 2016).

Tal y como recoge Isorna (2016), son muchas las instituciones e investigaciones que han venido mostrando en los últimos años una creciente preocupación por los adolescentes con TDAH, muchas de ellas con cierta controversia. En un meta-análisis realizado por Groenman, Janssen y Oosterlaan (2017) indican que es más frecuente el consumo de drogas en los individuos diagnosticados con TDAH que en sus iguales sin el diagnóstico del trastorno. El consumo de tabaco en adolescentes con TDAH es especialmente importante, ya que el retraso en su edad de inicio disminuye el consumo de otras drogas en el futuro (Lee, Mclernon, Kollins, \& Fuemmeler, 2018). En cuanto al consumo de cannabis hay estudios que defienden una mejora de los síntomas del trastorno gracias a su consumo (Cooper et al., 2017; Mitchell, Sweitzer, Tunno, Kollins, \& McClernon, 2016), mientras que otros llegan a conclusiones totalmente opuestas (Apple, Greydanus, \& Merrick, 2016), incluyendo el desarrollo de trastornos psicóticos en muchos de los consumidores (Di Forti et al., 2015). Asimismo, la literatura reporta un inicio más temprano del consumo de alcohol, tabaco, cannabis y otras sustancias entre los adolescentes con TDAH frente a sus pares no diagnosticados (Isorna, Golpe, Requeijo, Ayesta, \& Gómez, 2018; Molina et al., 2018). Son abundantes los trabajos que correlacionan el consumo de cocaína con el TDAH, indicándose que hasta un 20.5\% de sus consumidores cumple los criterios diagnósticos del mismo (Pérez de los Cobos et al. 2011). Sin embargo, si bien existen trabajos en los últimos años que analizan el consumo de drogas y TDAH (Miranda et al., 2016; Ochoa, Madoz, Villacieros, Llama, \& Sancho, 2010; Ortiz, Díaz, 
Cornejo, \& Sierra, 2017), la gran mayoría de estos estudios se basan en muestras clínicas, los cuales no estudian a estos sujetos comparándolos con sus compañeros extraídos de su mismo contexto y área geográfica y mismo centro educativo y no tienden a establecer perfiles diferenciales entre hombres y mujeres. En esta línea, varios estudios han llegado a conclusiones diversas, aunque Ottosen, Petersen, Larsen, \& Dalsgaard (2016) han hallado mayores tasas de abuso de alcohol y cannabis en las mujeres con respecto a los hombres con TDAH en una muestra de casi 20000 sujetos con TDAH.

En consecuencia, el objetivo principal del presente trabajo es establecer la prevalencia y patrón de consumo de drogas legales e ilegales en adolescentes (de 12 a 18 años) con diagnóstico de TDAH frente a sus pares sin diagnóstico. Asimismo, se desarrollan la siguiente serie de objetivos específicos: (a) evaluar los hábitos de consumo de tabaco y consumo de riesgo de alcohol, cannabis, cocaína, éxtasis, anfetaminas y alucinógenos; (b) conocer la edad de inicio de consumo de las distintas sustancias; (c) explorar e identificar un perfil diferencial entre hombres y mujeres indagando posibles diferencias.

\section{Método}

\section{Participantes}

Para dar cuenta de los objetivos planteados se recurrió a una metodología selectiva $o$ correlacional. El trabajo se enmarcaba dentro de un estudio más amplio, bajo un convenio de colaboración entre la Universidad de Santiago y el municipio de Santiago de Compostela. Se utilizó un muestreo intencionado, contactando con 12 centros escolares (de titularidad pública o concertada) que imparten estudios de Educación Secundaria Obligatoria (ESO) y Bachillerato en la ciudad de Santiago de Compostela y área metropolitana. Accedieron a participar en el estudio la totalidad de los centros contactados.

El número inicial de cuestionarios fue de 3431, si bien 11 fueron eliminados debido a patrones de respuesta incoherentes (con incongruencias manifiestas entre apartados relacionados del cuestionario), 19 por valores missing y 62 por encontrarse fuera del rango de edad objeto de estudio (entre los 12 y los 18 años). La muestra final estuvo comprendida por 3339 alumnos $(\mathrm{M}=14.60 ; D E=1.789)$, con una distribución por género de $50.1 \%$ de mujeres y $49.9 \%$ de hombres. En cuanto a la distribución por cursos, el $38.9 \%$ cursaban $1^{\circ}$ y $2^{\circ}$ de la ESO, el $33.2 \%$ cursaban $3^{\circ}$ y $4^{\circ}$ de la ESO y el $27.9 \%$ Bachillerato. De entre todo el alumnado de los centros, 222 adolescentes $(6.65 \%$ de la muestra total) han sido diagnosticados con TDAH por el sistema sanitario.

Tabla 1. Variables sociodemográficas

\begin{tabular}{lccc}
\hline & & $\begin{array}{c}\text { Con TDAH } \\
(\mathrm{n}=222)\end{array}$ & $\begin{array}{c}\text { Sin TDAH } \\
(\mathrm{n}=3117)\end{array}$ \\
\hline \multirow{2}{*}{ Género } & Hombre & $68 \%(\mathrm{n}=151)$ & $48.6 \%(\mathrm{n}=1515)$ \\
& Mujer & $32 \%(\mathrm{n}=71)$ & $51.4 \%(\mathrm{n}=1602)$ \\
Edad & & 14.91 años & 14.58 años \\
& & $36 \%$ & $38.9 \%$ \\
Curso & $1^{\mathrm{o}}{\mathrm{y} 2^{\circ} \text { ESO }}^{\mathrm{a}} \mathrm{y} \mathrm{4}^{\mathrm{o}}$ ESO & $41 \%$ & $32.6 \%$ \\
& Bachillerato & $23 \%$ & $28.5 \%$ \\
\hline
\end{tabular}

\section{Instrumentos}

Los datos fueron recogidos mediante un cuestionario ad hoc, diseñado expresamente para este estudio, en el que se incluían preguntas agrupadas en 3 bloques:

(1) Un primer bloque integrado por preguntas acerca de los hábitos de consumo de drogas legales e ilegales extraídos del ESTUDES 20142015 (Plan Nacional Sobre Drogas, 2016). Estas serían si alguna vez en el último año y en el último mes ha bebido alcohol, ha tomado 3 consumiciones en un mismo día, ha tomado 6 consumiciones en un mismo día, se ha emborrachado, ha fumado tabaco, ha consumido marihuana o hachís, cocaína y éxtasis, anfetaminas o alucinógenos.

(2) Un segundo bloque que incluye las herramientas de screening para la detección del consumo de riesgo de alcohol, tabaco, cannabis y otras drogas ilegales.

El Alcohol Use Disorders Identification Test (AUDIT) en su versión autoadministrada (Rial, Golpe, Araujo, Braña y Varela 2017) para estimar el consumo de riesgo de alcohol. Este test consta de 10 ítems que evalúan la cantidad y frecuencia del consumo (ítems 1-3), los posibles síntomas de dependencia (ítems 4-6) y los problemas relacionados con el consumo de alcohol (ítems 710). El formato de respuesta es tipo Likert, que va 
desde $0=$ "Nunca" hasta $4=$ " 4 o más veces a la semana" para el ítem 1; desde $0=$ "Nunca" hasta 4="Todos o casi todos los días" desde el ítem 2 hasta el 8; y desde $0=$ "Nunca" hasta $4=$ "Sí, en el último año" para los ítems 9 y 10. Los ocho primeros ítems se puntúan de 0 a 4 (5 categorías ordinales) y los ítems 9 y 10 con 0,2 y 4 puntos ( 3 categorías). La puntuación global puede oscilar entre 0-40 y, tendiendo estrictamente al punto de corte recomendado por los autores (puntuación “4”), es posible identificar un consumo de riesgo. La consistencia interna que presenta en el presente trabajo resultó aceptable $(\alpha=.84)$ e incluso algo mayor que la encontrada en el estudio de validación de Rial et al. (2017) $(\alpha=.82)$

La versión del CRAFFT Abuse Screening Test traducida al español y validada por Rial, et al. (2019) Se trata de un test de 6 ítems destinado a evaluar el consumo de riesgo de drogas en general. El formato de respuesta es dicotómico $(0=$ "No"; 1="Sí") y la puntuación global puede oscilar entre 0 y 6 , considerándose como consumo de riesgo una puntuación superior igual a " 2 ". La consistencia interna de la escala obtenida en el presente trabajo fue ligeramente inferior a la obtenida en la validación española ( $\alpha=.72$ vs. $\alpha=.74$ )

La subescala de Uso y Abuso de Sustancias del Problem Oriented Screening Instrument for Teenagers (POSIT ${ }_{\mathrm{UAS}}$ ) validada a la población española por Araujo, Golpe, Braña, Varela y Rial (2017). Se trata de una herramienta destinada a evaluar el consumo de riesgo de drogas en general con un número total de 17 ítems. El formato de respuesta es dicotómico ( $0=$ "No"; $1=$ "Sín") y la puntuación global puede oscilar entre 0 y 17 . El punto de corte recomendado por los autores es una puntuación igual o superior a 2. Esta subescala muestra una elevada consistencia interna con un $\alpha=84$, ligeramente superior a la obtenida en la validación española $(\alpha=.82)$.

La herramienta Cannabis Abuse Screening Test (CAST) (Legleye, Piontek, Kraus, Morand, \& Falissard, 2013), permite detectar un consumo de riesgo de cannabis. Consta de 6 ítems con un formato de respuesta tipo Likert que va desde $0=$ "Nunca" hasta 4="Muy a menudo" (5 categorías ordinales). La puntuación global puede oscilar entre 0 y 24 y el punto de corte utilizado es una puntuación igual o superior a 4, cuya consistencia interna resultó elevada $(\alpha=.87)$, superior a la obtenida en el trabajo de validación en España, llevado a cabo por Cuenca-Royo et al. (2012) $(\alpha=.75)$.

(3) Un último apartado en el que se recoge información sobre variables sociodemográficas, como el género, la edad, el curso académico actual y para el diagnóstico de TDAH se ha preguntado si el adolescente ha sido diagnosticado con TDAH por el sistema sanitario, incluyendo todos los subtipos.

\section{Procedimiento}

Los datos fueron recogidos en las propias aulas de los centros, en grupos reducidos (entre 15 y 25 individuos), mediante un cuestionario que cada estudiante ha cumplimentado de manera individual. Durante la recogida de datos un equipo de psicólogos con experiencia acreditada en la realización de este tipo de tareas informó de la confidencialidad, anonimato, voluntariedad y finalidad del estudio. El tiempo de cumplimentación del cuestionario fue de aproximadamente 20-25 minutos. Se contó con el consentimiento y la colaboración tanto de la dirección de los centros, como de las respectivas asociaciones de madres y padres de alumnos (AMPAs). El trabajo contó además con la aprobación del Comité de Bioética de la Universidad de Santiago de Compostela (Ref. 024/2018).

\section{Análisis de datos}

Se realizó una tabulación bivariada, con la aplicación de pruebas $t$ de Student para la comparación de medias y contrastes $\chi^{2}$ para la comparación de porcentajes. Asimismo, se halló el estadístico $V$ de Cramer para estimar el tamaño del efecto en las variables categóricas y del coeficiente Eta $(\eta)$ para las variables de intervalo o razón. De cara a garantizar un uso adecuado de pruebas paramétricas como la " $t$ " de Student, fueron comprobados previamente el supuesto de normalidad (mediante la prueba K-S con corrección de Lilliefors) y el supuesto de homocedasticidad u homogeneidad de varianzas (mediante la prueba de Levene). Dado el incumplimiento de ambos supuestos, especialmente el de normalidad, se optó por aplicar de manera complementaria la prueba de 
Tabla 2. Hábitos de consumo de sustancias psicoactivas

\begin{tabular}{|c|c|c|c|c|}
\hline & & Con TDAH (\%) & Sin TDAH $(\%)$ & $\chi^{2}$ \\
\hline \multirow{2}{*}{ Has bebido alcohol } & Último año & 58.1 & 48.4 & $7.388^{*}$ \\
\hline & Último mes & 36 & 26.5 & $9.070 *$ \\
\hline \multirow{2}{*}{ Emborracharse } & Último año & 28.8 & 27.4 & 0.145 \\
\hline & Último mes & 16.7 & 12.2 & 3.340 \\
\hline \multirow{2}{*}{ Has tomado 3 consumiciones en un mismo día } & Último año & 35.6 & 31.3 & 1.572 \\
\hline & Último mes & 22.5 & 16 & $5.994 *$ \\
\hline \multirow{2}{*}{ Has tomado 6 consumiciones en un mismo día } & Último año & 22.1 & 16.4 & $4.420^{*}$ \\
\hline & Último mes & 12.6 & 7 & $8.660 *$ \\
\hline \multirow{2}{*}{ Has fumado tabaco } & Último año & 38.7 & 25.4 & $18.213 * *$ \\
\hline & Último mes & 24.3 & 15.9 & $10.040 *$ \\
\hline \multirow{2}{*}{ Has consumido marihuana o hachís } & Último año & 22.1 & 15.3 & $6.600^{*}$ \\
\hline & Último mes & 12.2 & 8.3 & 3.540 \\
\hline \multirow{2}{*}{ Has consumido cocaína } & Último año & 1.8 & 1.4 & 0.046 \\
\hline & Último mes & 0.5 & 0.6 & 0.001 \\
\hline \multirow{2}{*}{$\begin{array}{l}\text { Has consumido éxtasis, anfetaminas o } \\
\text { alucinógenos }\end{array}$} & Último año & 0.5 & 1.9 & $7.840^{*}$ \\
\hline & Último mes & 0.9 & 0.6 & 0.004 \\
\hline
\end{tabular}

Mann-Whitney, cuyo uso es recomendado en este tipo de circunstancias (Rial \& Varela, 2008). Los análisis fueron realizados con el paquete estadístico IBM SPSS Statistics 25.

\section{Resultados}

\section{Diagnóstico de TDAH}

Tal y como se muestra en la Tabla 1, el 6.65\% del alumnado $(n=222)$ informa que presenta un diagnóstico de TDAH por el sistema sanitario, siendo el $68 \%$ varones $(n=151)$ y el $32 \%$ mujeres $(\mathrm{n}=71)$.

\section{Hábitos de consumo}

Tal y como se puede observar en la Tabla 2, el consumo de sustancias psicoactivas es, en general, mayor en los adolescentes con TDAH frente a sus pares sin su diagnóstico. El $58.1 \%$ de los adolescentes con TDAH informan de haber bebido alcohol en el último año, frente al $48.4 \%$ de los adolescentes sin TDAH. No se han encontrado diferencias estadísticas en el hábito de emborracharse. Con respecto al binge drinking, cabe señalar que se ha encontrado un porcentaje superior de adolescentes con TDAH tanto en el último año ( $22.1 \%$ vs $16.4 \%$ ), como en el último mes (12.6\% vs $7 \%)$. El tabaco es la sustancia que muestra mayores diferencias en el consumo en el último año que realizan los adolescentes con TDAH frente a los que no están diagnosticados ( $38.7 \%$ vs $25.4 \%$ ). Asimismo, el $22.1 \%$ de los adolescentes con TDAH informan haber consumido marihuana o hachis frente al $15.3 \%$ de sus iguales sin el trastorno. Entre las demás sustancias estudiadas, cabe señalar que la cocaína no ha mostrado diferencias significativas entre los grupos de la muestra, mientras que se evidencian diferencias en el consumo de éxtasis, anfetaminas $\mathrm{o}$ alucinógenos en el último año (5\% vs $1.9 \%$ ).

Los datos recogidos en las Tablas 3 y 4 revelan diferencias en los hábitos de consumo de los hombres y mujeres con y sin TDAH tanto en el último año como en el último mes. Los hombres con TDAH muestran una tasa superior en el consumo de tabaco y de éxtasis, anfetaminas o alucinógenos en el último año. Sin embargo, en el caso de las mujeres se han hallado tasas más elevadas de consumo de alcohol, binge drinking, consumo de tabaco y consumo de cannabis tanto en el último año como en el último mes. Aunque en todos los casos se obtienen tamaños de efecto pequeños, sí es relevante la diferencia encontrada según el género de los sujetos. Así, el TDAH en hombres no muestran relación ninguna en el consumo de alcohol, CIA, consumo de cannabis, cocaína y éxtasis, anfetaminas o alucinógenos tanto en el último año como en el último mes. En el caso de las mujeres se han encontrado correlaciones pequeñas y positivas en el consumo de todas las drogas salvo en el de la de cocaína y el éxtasis, las anfetaminas o alucinógenos en el último año y en el último mes. Con respecto al tabaco, en el caso de los hombres se encuentra un tamaño del efecto pequeño $(V=.065)$ en su consumo en el último año y no significativo en el último mes, mientras que para las mujeres se obtiene un tamaño de efecto significativo en ambos 
Tabla 3. Hábitos de consumo de sustancias psicoactivas

\begin{tabular}{|c|c|c|c|c|c|c|c|c|c|}
\hline \multicolumn{2}{|c|}{ Último año } & \multicolumn{4}{|c|}{ Hombres } & \multicolumn{4}{|c|}{ Mujeres } \\
\hline & & $\begin{array}{l}\text { Con } \\
\text { TDAH } \\
(\%)\end{array}$ & $\begin{array}{l}\text { Sin } \\
\text { TDAH } \\
(\%)\end{array}$ & $\chi^{2}$ & $V$ & $\begin{array}{l}\text { Con } \\
\text { TDAH } \\
(\%)\end{array}$ & $\begin{array}{l}\text { Sin } \\
\text { TDAH } \\
(\%)\end{array}$ & $\chi^{2}$ & $V$ \\
\hline Has b & do alcohol & 53 & 46.6 & 2.007 & --- & 70 & 50.1 & $9.805^{*}$ & .081 \\
\hline \multirow{3}{*}{ CIA } & Emborracharse & 20.8 & 26 & 1.658 & --- & 47.1 & 28.7 & $10.101 * *$ & .082 \\
\hline & $\begin{array}{l}3 \text { consumiciones en } \\
\text { un mismo día }\end{array}$ & 27.5 & 30.8 & 0.525 & --- & 52.9 & 31.7 & $12.689 * *$ & .092 \\
\hline & $\begin{array}{l}6 \text { consumiciones en } \\
\text { un mismo día }\end{array}$ & 17.4 & 17.5 & 0.001 & --- & 32.9 & 15.2 & $14.255^{* *}$ & .098 \\
\hline \multirow{2}{*}{\multicolumn{2}{|c|}{$\begin{array}{l}\text { Has fumado tabaco } \\
\text { Has consumido marihuana o } \\
\text { hachís }\end{array}$}} & 32.2 & 22.6 & $6.426^{*}$ & .065 & 54.3 & 28 & $21.197 * *$ & .117 \\
\hline & & 19.5 & 16.8 & 0.518 & --- & 28.6 & 13.9 & $10.401 * *$ & .084 \\
\hline \multicolumn{2}{|c|}{ Has consumido cocaína } & 2 & 1.7 & 0.001 & --- & 1.4 & 1.1 & 0.001 & --- \\
\hline \multicolumn{2}{|c|}{$\begin{array}{l}\text { Has consumido éxtasis, } \\
\text { anfetaminas o alucinógenos }\end{array}$} & 6 & 2.6 & $4.308^{*}$ & .058 & 2.9 & 1.2 & 0.504 & --- \\
\hline
\end{tabular}

Nota. ${ }^{*} p<.05 ; * * p<.001 ; \chi^{2}$ : Chi cuadrado; V: V de Cramer

Tabla 4. Hábitos de consumo de sustancias psicoactivas

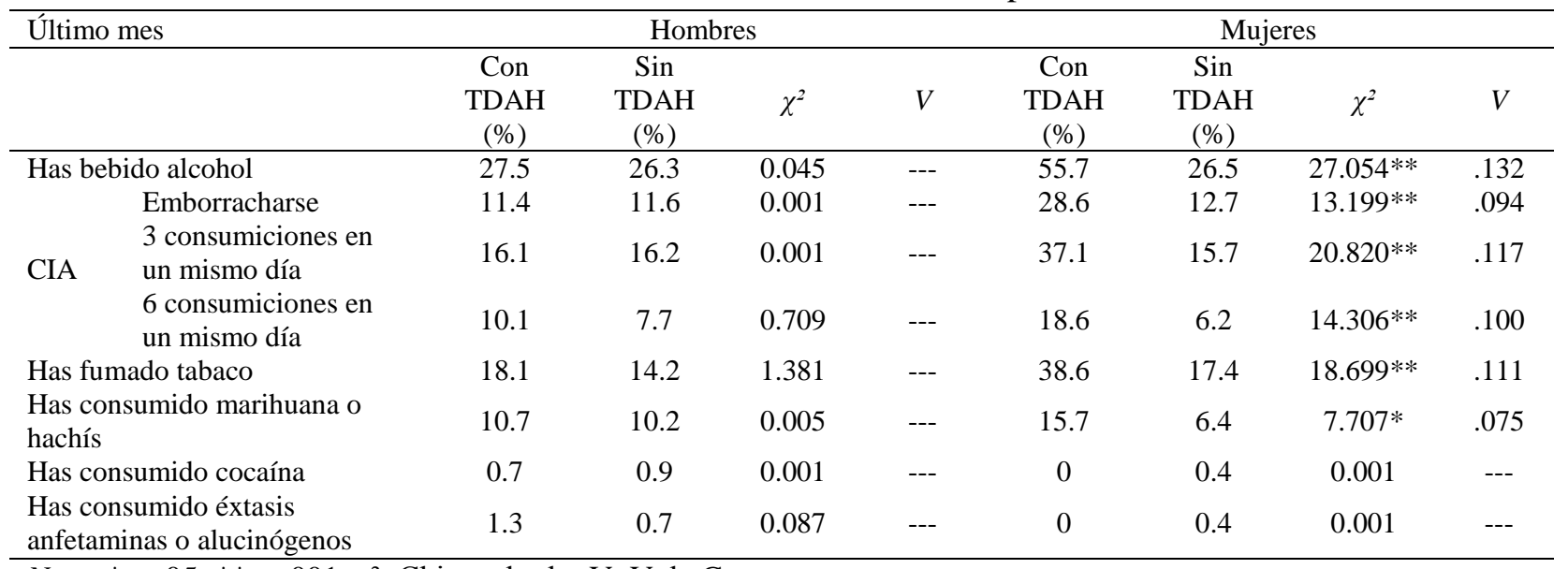

Nota. ${ }^{*} p<.05 ; * * p<.001 ; \chi^{2}$ : Chi cuadrado; V: V de Cramer

casos ( $V=.117$ para el último año y $V=.111$ para el último mes). Tal y como establece Cohen (1988), la interpretación de la $\mathrm{V}$ de Cramer es similar a la de una correlación de Pearson, por lo que las asociaciones encontradas deben considerarse, en cualquier caso, de baja magnitud.

Tal y como se muestra en la Tabla 5, los resultados del screening ponen de manifiesto que los adolescentes con TDAH muestran una tasa mayor de consumo de riesgo de alcohol (AUDIT), de cannabis (CAST) y de drogas en general (CRAFFT). No obstante, al analizar estas diferencias segmentando la muestra por el género de los participantes, se encuentran diferencias principalmente en las mujeres que han sido diagnosticadas con TDAH frente a sus pares. Las mujeres con TDAH muestran casi el doble de consumo de riesgo de alcohol (AUDIT: $47.1 \%$ vs $24.4 \%$ ), más del triple de consumo de riesgo de cannabis (CAST: $11.3 \%$ vs $5.2 \%$ ), así como tasas significativamente superiores en el consumo de riesgo de drogas en general (CRAFFT: $38.6 \%$ vs $22.4 \%$ y POSIT: $47.1 \%$ vs $32.9 \%$ ). Los tamaños de efecto encontrados revelan cómo el TDAH es una variable que se relaciona con el consumo de riesgo con un tamaño de efecto reducido, pero de manera diferente según el género. Estas diferencias se pueden apreciar sobre todo en el consumo de riesgo de alcohol y de cannabis. Mientras que el TDAH en los hombres no implica diferencias significativas, sí lo es en el caso de las mujeres.

\section{Edad de inicio}

Tal y como se puede apreciar en la Figura 1, los adolescentes con TDAH comienzan a consumir desde edades más tempranas en todas las sustancias, si bien estas diferencias son únicamente significativas en el caso del tabaco (13.36 vs 13.93$)$ y el cannabis (14.45 vs 14.90$)$. 
Tabla 5. Consumo de riesgo de alcohol, cannabis y drogas en general

\begin{tabular}{ccccc}
\hline & Con TDAH $(\%)$ & Sin TDAH $(\%)$ & $\chi^{2}$ & V \\
\hline AUDIT & 32.7 & 24.3 & $7.740^{*}$ & .049 \\
Hombre & 26.6 & 24.2 & 0.287 & --- \\
Mujer & 47.1 & 24.4 & $17.199^{* *}$ & .106 \\
CAST & 11.3 & 5.2 & $13.420^{* *}$ & .067 \\
Hombre & 10.1 & 6.3 & 2.493 & --- \\
Mujer & 14.3 & 4 & $14.103^{* *}$ & .100 \\
CRAFFT & 28.6 & 21.5 & $5.615^{*}$ & .043 \\
Hombre & 24.5 & 20.5 & 1.040 & ---- \\
Mujer & 38.6 & 22.4 & $8.932^{*}$ & .078 \\
POSIT & 35.3 & 30.7 & 1.825 & --- \\
Hombre & 30.2 & 28.4 & 0.140 & --- \\
Mujer & 47.1 & 32.9 & $5.510^{*}$ & .061 \\
\hline
\end{tabular}

Nota. ${ }^{*} p<.05 ; * * p<.001 ; \chi^{2}$ : Chi cuadrado; V: V de Cramer

Tabla 6. Edad de inicio sustancias psicoactivas segmentado por género

\begin{tabular}{|c|c|c|c|c|c|c|}
\hline & & Con TDAH $(\overline{\mathrm{X}})$ & Sin TDAH $(\bar{X})$ & $\mathrm{t}$ & $\mathrm{Z}$ & $\eta$ \\
\hline \multirow{2}{*}{ Alcohol } & Hombre & 13.43 & 13.61 & 0.773 & -1.055 & --- \\
\hline & Mujer & 13.77 & 13.94 & 0.758 & -0.310 & --- \\
\hline \multirow{2}{*}{ Tabaco } & Hombre & 12.76 & 13.75 & $2.813^{*}$ & $-2.865^{*}$ & 0.226 \\
\hline & Mujer & 14.20 & 14.09 & -0.403 & -1.039 & --- \\
\hline \multirow{2}{*}{ Cannabis } & Hombre & 14.44 & 14.74 & 1.224 & -1.319 & --- \\
\hline & Mujer & 14.48 & 15.07 & $2.814 *$ & -1.831 & 0.308 \\
\hline \multirow{2}{*}{ Cocaína } & Hombre & 16.5 & 15.81 & -0.696 & -0.729 & --- \\
\hline & Mujer & 14 & 15.63 & 1.950 & -1.879 & --- \\
\hline
\end{tabular}

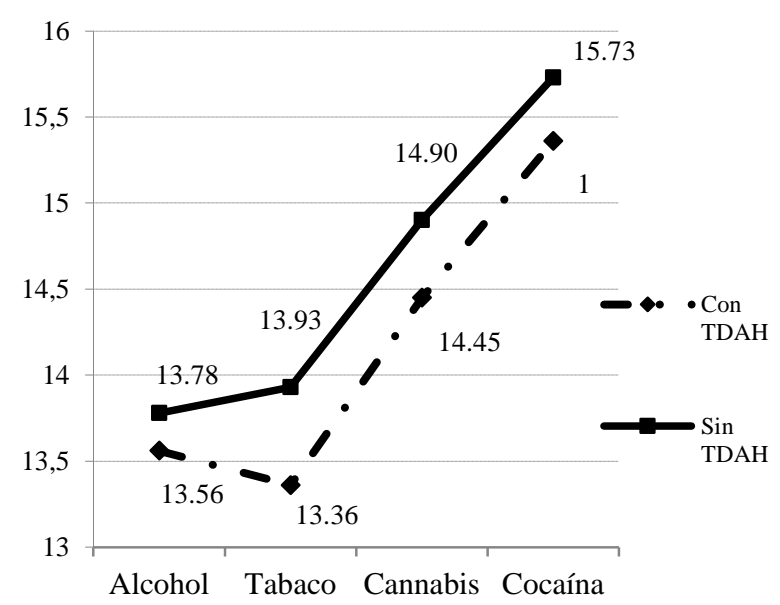

Figura 1. Edad de inicio de las sustancias psicoactivas

Tal y como se muestra en la Tabla 6, tras analizar diferencialmente las edades de inicio según el género se ha encontrado que los hombres con TDAH comienzan a fumar casi un año antes que sus pares sin el trastorno (12.76 vs 13.75). Las mujeres con TDAH, por su parte, inician el consumo de cannabis casi medio año antes que las no diagnosticadas (14.48 vs 15.07 ).

\section{Discusión y Conclusiones}

En cuanto al primer objetivo del estudio, la prevalencia global de TDAH registrado fue del $6.65 \%$, siendo diagnosticado más del doble en varones que en mujeres ( $68 \%$ vs $32 \%$ ), porcentaje que se ajustan a lo referenciado en otros estudios realizados en España (Catala-López et al., 2012; Isorna et al., 2017; Rivas et al., 2017). También se han confirmado tasas mayores de consumo de tabaco, alcohol, cannabis, éxtasis, anfetaminas y alucinógenos en los adolescentes con diagnóstico de TDAH frente a sus pares sin el trastorno, como ya han advertido otros autores (Groenman et al., 2017; van Emmerik-van Oortmerssen et al., 2012; Isorna, Golpe, Otero, Ayesta, \& Gómez, 2018; Miranda et al., 2016). No obstante, estas diferencias, aunque importantes, están lejos de las encontradas por otros trabajos donde se indican tasas de consumo hasta tres veces mayores en los adolescentes con TDAH (Gudjonsson, Sigurdsson, Sigfusdottir y Young, 2012). Asimismo, al segmentar los datos por el género de los sujetos, se ha podido comprobar que, aunque los hombres con TDAH tienen consumos ligeramente superiores, son principalmente las mujeres con TDAH las que presentan unas tasas de consumo más relevantes. Así, mientras que en las mujeres las tasas de consumo alcohol, CIA, tabaco y cannabis se duplican, no se han hallado diferencias significativas entre los hombres. Estos resultados diferentes según el género del consumidor difieren de los encontrados en otros 
estudios (Babinski et al., 2011; Biederman, Petty, Hammerness, Batchelder, \& Faraone, 2012) y parecen semejarse a los publicados recientemente por Elkins et al. (2018).

Pese a que la literatura ha evidenciado un mayor consumo de cocaína en los individuos diagnosticados con TDAH (Liebrenz, Gamma, Ivanov, Buadze, \& Eich, 2016), en el presente estudio no se han encontrado diferencias estadísticamente significativas al respecto. No obstante, se debe tener en cuenta que la edad de inicio media del consumo de cocaína en polvo y/o base se encuentra en 21.1 años en la población española según el EDADES 2017-2018 (Plan Nacional Sobre Drogas, 2019), la cual se encuentra fuera del rango de edad objeto del presente estudio.

Para conocer el patrón de consumo de los adolescentes con TDAH no es suficiente con evaluar su frecuencia y/o hábitos de consumo, sino que también es necesario conocer sus niveles de consumo de riesgo, en nuestro caso medidos a través del CRAFT y POSIT. En este sentido, se ha podido comprobar que los adolescentes con TDAH presentan un mayor consumo de tabaco, así como un consumo de riesgo mayor de alcohol, cannabis y éxtasis, anfetaminas o alucinógenos, tal y como muestran otros trabajos (Riegler, Völkl-Kernstock, Lesch, \& Henriette, 2017). Sin embargo, se ha vuelto a constatar que las diferencias en el CRAFFT y POSIT se manifiestan exclusivamente en las mujeres que presentan el trastorno ya que el consumo de riesgo hallado en los hombres con TDAH no muestra diferencias con su propio grupo natural de referencia. En esta línea, Ottosen et al. (2016) indican que en el imaginario social existe el estereotipo de que las mujeres con TDAH tienen menos problemas asociados a un consumo abusivo de drogas, pero en realidad tienen un riesgo mayor de sufrir un trastorno por consumo de sustancias.

En cuanto al segundo objetivo, el estudio de las edades de inicio de consumo de las distintas sustancias evaluadas, la literatura encontrada advierte que las mayores diferencias se encuentran en el inicio de consumo de tabaco (Molina et al., 2018; Isorna et al., 2018). En esta misma línea Martínez-Raga y Knecht (2012) plantean que el consumo temprano de alcohol o tabaco es predictivo del posterior riesgo de desarrollar problemas por consumo de otras sustancias en sujetos con TDAH, siendo el tabaco la sustancia con mayor valor predictivo para desarrollar un trastorno por uso de sustancias. El presente estudio pone de manifiesto que los varones con TDAH comienzan a consumir tabaco un año antes de lo que lo hacen sus pares sin el diagnóstico, mientras que en las mujeres no se han hallado diferencias. Sin embargo, esta situación se invierte en el consumo de cannabis, siendo las mujeres con TDAH las que se inician a una menor edad. En las demás sustancias, las edades de inicio son semejantes entre los dos grupos analizados. En relación a esto se debe hacer hincapié en la importancia de la detección precoz y la importancia del tratamiento a edades tempranas, ya que los niños/as con un TDAH no tratado tienen más riesgo de desarrollar una adicción que los niños/as sin este trastorno, tienden a iniciarse precozmente en el consumo de sustancias y pasan más rápidamente del uso, al abuso y a la dependencia de sustancias (Daigre, et al., 2013). En definitiva, y en relación al tercer objetivo del presente estudio, se ha puesto de manifiesto que para evaluar el consumo de sustancias en adolescentes con TDAH es importante tener en cuenta el consumo diferencial por género. Algunos autores apuntan como causa de estas diferencias el hecho de que las jóvenes son diagnosticadas más tarde con TDAH y, por lo tanto, acceden más tarde al tratamiento (Ottosen et al., 2016). En este sentido, el acceso temprano al tratamiento de TDAH parece ser un factor de protección en el desarrollo de un trastorno por consumo de sustancias; ello puede deberse a que los principios activos de los fármacos, principalmente el metilfenidato, están contraindicados con el consumo de drogas.

Sin embargo, tambien se han barajado otros posibles factores que pueden explicar las diferencias encontradas por género. Por un lado, se ha asociado tradicionalmente a los chicos con TDAH con "tener un comportamiento más disruptivo" mientras que la hiperactividad en las chicas tiende a percibirse como inusual e incluso como extraña, siendo más predominante el déficit de atención (Hjörne \& Evaldsson, 2015). Por otro lado, tradicionalmente se ha constatado que los progenitores se involucran más en actividades 
sociales con niños que con niñas temperamentalmente difíciles (Parke, Power, Tinsley, \& Hymel, 1981). Así pués, las diferencias en el papel de los progenitores según el género de sus hijos puede ser otro factor que incide en las diferencias encontradas. En este estudio se ha intentado explorar también el papel del género en el consumo de sustancias en adolescentes con TDAH, evidenciando interesantes diferencias, que deben ser analizadas en futuros trabajos con mayor profundidad y bajo una perspectiva de género.

\section{Limitaciones}

En cuanto a las limitaciones del trabajo, debe referirse en primer lugar a la naturaleza trasversal del mismo. Debido a ello, no es posible establecer relaciones de causalidad entre las variables objeto de estudio. Por otro lado, a pesar de utilizar una muestra con más de 3000 adolescentes, el hecho de utilizar un muestreo no probabilístico seleccionando sujetos pertenecientes únicamente a Santiago de Compostela y zonas limítrofes, limita la validez externa de los resultados. No obstante, es obvio que futuros trabajos han de marcarse como objetivo investigar el consumo de drogas en escolares diagnosticados con TDAH frente a sus iguales sin diagnóstico en otras comunidades autónomas. Asimismo, el hecho de que los datos hayan sido recogidos en el contexto escolar y no en los servicios de Atención Primaria o pediatría, hace que las variables analizadas hayan sido autoinformadas, por lo que es imposible conocer a ciencia cierta en qué medida los adolescentes pueden realmente haber infraestimado o sobreestimado sus niveles de consumo y el propio diagnóstico de TDAH. Sin embargo, otros expertos en conductas adictivas ya han establecido que las medidas autoinformadas anónimas y confidenciales han demostrado ser fiables e incluso mejores que otros métodos a la hora de evaluar los niveles de consumo de drogas (Babor, Kranzler, \& Lauerman, 1989; Winters, Stinchfield, Henly, \& Schwartz, 1990).

Por último, dado el reducido tamaño muestral y la consecuente dificultad para controlar estadísticamente el posible sesgo derivado de la diferente composición de los grupos por género, se han realizado todos los análisis estadísticos de forma separada, para mujeres y para hombres, comprobando la existencia de importantes diferencias. Sin duda, ello constituye una limitación del trabajo pero, al mismo tiempo, una interesante línea de investigación para el futuro.

\section{Reconocimientos}

Los autores de este trabajo quieren agradecer la financiación recibida a través de la Delegación del Gobierno para el Plan Nacional sobre Drogas (Ref. 2018/008) para la realización de este estudio.

\section{Conflicto de intereses}

Los autores de este artículo declaran no tener ningún conflicto de intereses.

\section{Referencias}

Apple, R. W., Greydanus, D. E., \& Merrick , J. (2016). Smoking cannabis is especially dangerous for youth diagnosed with attention deficit/hyperactivty disorer (ADHD). Journal of Community Medicine y Health Education, 6(4), 451-453. doi:10.4172/2161-0711.100045 Araujo, M., Golpe, S., Braña, T., Varela, J., \& Rial, A. (2018). Validación psicométrica del POSIT para el cribado del consumo de riesgo de alcohol y otras drogas entre adolescentes. Adicciones, 30(2), 130-139. doi:10.20882/adicciones.958

Asociación Americana de Psiquiatría [APA]. (2014). Manual diagnóstico y estadístico de los trastornos mentales ( $5^{\circ}$ ed.) [DSM-5]. Madrid: Editorial Médica Panamericana.

Asociación Proyecto Hombre (2016). Observatorio Proyecto Hombre sobre el perfil de las personas con problemas de adicción en tratamiento. Madrid, España: Asociación Proyecto Hombre.

Babinski, D. E., Pelham, W. E., Molina, B. S., Gnagy, E., Waschbusch, D. A., Yu, J., ... \& Karch, K. M. (2011). Late adolescent and young adult outcomes of girls diagnosed with ADHD in childhood: An exploratory investigation. Journal of Attention Disorders, 15(3), 204-214.

doi:10.1177/1087054710361586

Babor, T. F., Kranzler, H., \& Lauerman, R. (1989). Early detection of harmful alcohol 
consumption: Comparison of clinical, laboratory, and self-report screening procedures. Addictive Behaviors, 14, 139-157

Biederman, J., Petty, C. R., Hammerness, P., Batchelder, H., \& Faraone, S. V. (2012). Cigarette smoking as a risk factor for other substance misuse: 10-Year study of individuals with and without attention-deficit hyperactivity disorder. British Journal of Psychiatry, 201(3), 207-214. doi.org/10.1192/bjp.bp.111.100339

Bloemsma, J. M., Boer, F., Arnold, R., Banaschewski, T., Faraone, S. V., Buitelaar, J. K., Sergeant, J. A., Rommelse, N., \& Oosterlaan, J. (2013). Comorbid anxiety and neurocognitive dysfunctions in chil-dren with ADHD. European Child y Adolescent Psychiatry, 22, 225-234. doi:10.1007/s00787-012-0339-9

Carbia, C., López-Caneda, E., Corral, M., \& Cadaveira, F. (2018). A systematic review of neuropsychological studies involving young binge drinkers. Neuroscience and Biobehavioral Reviews, 90, 332-349. doi:10.1016/j.neubiorev.2018.04.013.

Catalá-López, F., Peiró, S., Ridao, M., SanfélixGimeno, G., Gènova-Maleras, R., \& Catalá, M.A. (2012). Prevalence of attention deficit hyperactivity disorder among children and adolescents in Spain: A systematic review and meta-analysis of epidemiological studies. BMC Psychiatry, 12, 1-13. doi:10.1186/1471-244X-12-168

Cohen, J. (1988). Statistical power analysis for the behavioral sciences. Second Edition. Hillsdate, NJ: LEA.

Cooper, R., Williams, E., Seegobin, S., Tye, C., Kuntsi, J., \& Asherson, P. (2017). Cannabinoids in attentiondeficit/hyperactivity disorder: A randomisedcontrolled trial. European Neuropsychopharmacology, 27, 795-808. doi.org/10.1016/j.euroneuro.2017.05.005

Cuenca-Royo, A. M., Sánchez-Niubó, A., Forero, C. G., Torrens, M., Suelves, J. M., \& Domingo-Salvany, A. (2012). Psychometric properties of the CAST and SDS scales in young adult cannabis users. Addictive Behaviors, 37(6), 709-715. doi.org/10.1016/j.addbeh.2012.02.012
Daigre, C., Terán, A., García-Vicent, V., \& Roncero, C. (2013). Trastorno por déficit de atención con hiperactividad y dependencia de sustancias depresoras. Una revisión. Adicciones, 25(2), 171-186. doi:http://dx.doi.org/10.20882/adicciones.65

Dalsgaard, S., Mortensen, P. B., Frydenberg, M., \& Thomsen, P. H. (2014). ADHD, stimulant treatment in childhood and subsequent substance abuse in adulthood: A naturalistic long-term follow-up study. Addictive Behaviors, 39(1), 325-328. doi:10.1016/j.addbeh.2013.09.002

Di Forti, M., Marconi, A., Carra, E., Fraietta, S., Trotta, A., Bonomo, M., ... \& Stilo, S. A. (2015). Proportion of patients in south London with first-episode psychosis attributable to use of high potency cannabis: A case-control study. The Lancet Psychiatry, 2(3), 233-238 doi.org/10.1016/S2215-0366(14)00117-5

Elkins, I. J., Saunders, G. R. B., Malone, S. M., Keyes, M. A., McGue, M., \& Iacono, W. G. (2018). Associations between childhood ADHD, gender, and adolescent alcohol and marijuana involvement: A causally informative design. Drug and Alcohol Dependence, 184, 33-41. doi:10.1016/j.drugalcdep.2017.11.011.

Golpe, S., Barreiro, C., Isorna, M., Varela, J., \& Rial, A. (2017). La práctica del botellón en adolescentes gallegos: Prevalencia, implicaciones y variables asociadas. Psicología Conductual, 25(3), 529-545.

González-Castro, P., Rodríguez, C., Cueli, M., Cabeza, L., \& Álvarez, L. (2014). Competencias matemáticas y control ejecutivo en estudiantes con Trastorno por Déficit de Atención con Hiperactividad y Dificultades de aprendizaje de las matemáticas. Revista de Psicodidáctica, 19(1), 125-143. doi:10.1387/RevPsicodidact.7510

Groenman, A. P., Janssen, T. W. P., \& Oosterlaan, J. (2017). Childhood psychiatric disorders as risk factor for subsequent substance abuse: A meta-analysis. Journal of the American Academy of Child y Adolescent Psychiatry, 56(7), 556-569. doi.org/10.1016/j.jaac.2017.05.004. 
Gudjonsson, G. H., Sigurdsson, J. F., Sigfusdottir, I. D., \& Young, S. (2012). An epidemiological study of ADHD symptoms among young persons and the relationship with cigarette smoking, alcohol consumption and illicit drug use. The Journal of Child Psychology and Psychiatry, 53(3), 304-3012. doi.org/10.1111/j.1469-7610.2011.02489.x

Hjörne, E., \& Evaldsson, A. C. (2015). Reconstituting the ADHD girl: Accomplishing exclusion and solidifying a biomedical identity in an ADHD class. International Journal of Inclusive Education, 19(6), 626-644.

doi:10.1080/13603116.2014.961685

Isorna, M. (2015). Trastorno por Déficit de Atención e Hiperactividad (TDAH). Santiago de Compostela: Andavira Ed.

Isorna, M. (2016). El niño hiperactivo y las polémicas en torno al diagnóstico y al tratamiento del TDAH. Innovación educativa, 26, 181-200.

Isorna, M., Rial, A., Felpeto, M., \& Mociño, L. R. (2017). Evaluación del impacto del efecto relativo de la edad en el rendimiento escolar, bullying, autoestima, diagnostico de TDAH y consumo de tabaco en el paso de educación primaria a secundaria. Revista Iberoamericana de Diagnóstico y Evaluación - e Avaliação Psicológica, 2(44), 92-104. doi.org/10.21865/RIDEP44.2.08

Isorna, M., Golpe, S., Otero, M., Ayesta, J., \& Gómez, P. (2018). Consumo de tabaco y autoestima en adolescentes con y sin Trastorno por Déficit de Atención con Hiperactividad (TDAH): Propuestas para una mejor prevención. Revista española de drogodependencias, 43(4), 69-82.

Lee, C. T., McClernon, F. J., Kollins, S. H., \& Fuemmeler, B. F. (2017). Childhood ADHD symptoms and future illicit drug use: The role of adolescent cigarette use. Journal of pediatric psychology, 43(2), 162-171. doi.org/10.1093/jpepsy/jsx098

Legleye, S., Piontek, D., Kraus, L., Morand, E., \& Falissard, B. (2013). Validation of the Cannabis Abuse Screening Test (CAST) using a latent class analysis of the DSM-IV among adolescents. International Journal of Methods in Psychiatric Research, 22(1), 16-26. doi:10.1002/mpr.1378.

Liebrenz, M., Gamma, A., Ivanov, I., Buadze, A., \& Eich, D. (2016). Adult attentiondeficit/hyperactivity disorder: Associations between subtype and lifetime substance use a clinical study. F1000Research, 4, 1-15. doi:10.12688/f1000research.6780.2

Martínez-Raga, J., \& Knecht, K. (2012). Patología Dual: Protocolos de intervención de TDAH. Madrid: Sociedad Española de Patología Dual.

Miranda, A., Colomer, C., Berenguer, C., Roselló, R., \& Roselló, B. (2016). Substance use in young adults with ADHD: Comorbidity and symptoms of inattention and hyperactivity/impulsivity. International Journal of Clinical and Health Psychology, 16(2), 157-165.

doi.org/10.1016/j.ijchp.2015.09.001

Mitchell, J., Sweitzer, M., Tunno, A., Kollins, S., \& McClernon J. (2016). "I use weed for my ADHD": A qualitative analysis of online forum discussions on cannabis use and ADHD. PLOS ONE, 11(5), 1-13. doi:10.1371/journal.pone.0156614.

Molina, B. S., Howard, A., Swanson, J., Stehli, A., Mitchell, J., Kennedy, T., ... \& Hoza, B. (2018). Substance use through adolescence into early adulthood after childhooddiagnosed ADHD: Findings from the MTA longitudinal study. Journal of Child Psychology and Psychiatry, 59(6), 692-702. https://doi.org/10.1111/jcpp.12855

Observatorio Europeo de las Drogas y las Toxicomanías. (2006). El problema de la drogodependencia en Europa. Luxemburgo, Gran Ducado de Luxemburgo: Oficina de Publicaciones Oficiales de las Comunidades Europeas.

Observatorio Español de las Drogas y las Adicciones [OEDA] (2020). Informe 2020. Alcohol, tabaco y drogas ilegales en España. Madrid: Delegación del Gobierno para el Plan Nacional sobre Drogas. Ministerio del Interior. Recuperado de: https://pnsd.sanidad.gob.es/profesionales/siste masInformacion/informesEstadisticas/pdf/202 0OEDA-INFORME.pdf

Ochoa, E., Madoz, A., Villacieros, I., Llama, P., \& Sancho, J. (2010). Trastorno por déficit de 
atención e hiperactividad (TDAH) y consumo de sustancias: Datos preliminares de seguimiento en una población de sujetos jóvenes. Trastornos Adictivos, 12(2), 79-86.

Organización Mundial de la Salud. (2004). Global status report on alcohol 2004. Génova, Suiza: Department of Mental Health and Substance Abuse.

Ortiz, B., Díaz, R., Cornejo, J., \& Sierra, G. A. (2017). Substance use disorder in patients with attention deficit hyperactivity disorder receiving stimulants. Acta Neurológica Colombiana, 33(4), 307-311.

Ottosen, C., Petersen, L., Larsen, J. T., \& Dalsgaard, S. (2016). Gender differences in associations between attentiondeficit/hyperactivity disorder and substance use disorder. Journal of the American Academy of Child y Adolescent Psychiatry, 55 (3), 227-234. doi:10.1016/j.jaac.2015.12.010

Parke, R. D., Power, T., Tinsley, B., \& Hymel, S. (1981). El papel del padre en el sistema familiar. Infancia y aprendizaje, 4(15), 39-51.

Pérez de los Cobos, J., Siñol, N., Puerta, C., Cantillano, V., López-Zurita, C., \& Trujols, J. (2011). Features and prevalence of patients with probable adult attention deficit hyperactivity disorder who request treatment for cocaine use disorders. Psychiatry Research, 185(1-2), 205-210. https://doi.org/10.1016/j.psychres.2009.03.019

Plan Nacional sobre Drogas. (2009). Estrategia Nacional sobre Drogas 2009-2016. Madrid, España: Ministerio de Sanidad y Consumo.

Plan Nacional sobre Drogas. (2016). Encuesta sobre el uso de drogas en enseñanzas secundarias en España (ESTUDES) 20142015. Madrid, España: Ministerio de Sanidad, Servicios Sociales e Igualdad

Plan Nacional sobre Drogas (2017). Encuesta sobre alcohol y otras drogas en España (EDADES) 2015-2016. Madrid, España: Ministerio de Sanidad, Servicios Sociales e Igualdad.

Plan Nacional sobre Drogas. (2017). Estrategia Nacional sobre Adicciones 2017-2024. Madrid, España: Ministerio de Sanidad y Consumo.

Parada, M., Corral, M., Caamaño-Isorna, F., Mota, N., Crego, A., Rodríguez Holguín, S.,
\& Cadaveira, F. (2011). Definición del concepto de consumo intensivo de alcohol adolescente (binge drinking). Adicciones, 23(1), 53-63.

doi:http://dx.doi.org/10.20882/adicciones.167

Quintero, J., Loro, M., Jiménez, B., \& GarcíaCampos, N. (2011). Aspectos evolutivos del Trastorno por Déficit de Atención e Hiperactividad (TDAH): De los factores de riesgo al impacto socioacadémico y a la comorbilidad. Vertex, 22, 101-108.

Reale, L., Bartoli, B., Cartabia, M., Zanetti, M., Costantino, M., Canevini, P, ... \& Bonati, M. (2017). Comorbidity prevalence and treatment outcome in children and adolescents with ADHD. European Child y Adolescent Psychiatry, 26(12). (Avance digital). https://doi.org/10.1007/s00787-017-1005-z

Rial, A., Golpe, S., Araujo, M., Braña, T., \& Varela, J. (2017). Validación del "test de identificación de trastornos por consumo de alcohol" (AUDIT) en población adolescente española. Psicología Conductual, 25 (2), 371386.

Rial, A., Golpe, S., Barreiro, C., Gómez, P., \& Isorna, M. (2020). La edad de inicio en el consumo de alcohol en adolescentes: Implicaciones y variables asociadas. Adicciones, 32(1), 52-62. doi:dx.doi.org/10.20882/adicciones.1266

Rial, A., Harris, S. K., Knight, J. R., Araujo, M., Gómez, P., Braña, T., \& Golpe, S. (2019). Validación empírica del CRAFFT Abuse Screening Test en una muestra de adolescentes españoles. Adicciones, 31(2), 160-169. doi.org/10.20882/adicciones. 1105

Rial, A., \& Varela, J. (2008). Estadística práctica para la investigación en ciencias de la salud. A Coruña: Netbiblo.

Riegler, A., Völkl-Kernstock, S., Lesch, O., Walter, H., \& Skala, K., (2017). Attention deficit hyperactivity disorder and substance abuse: An investigation in young Austrian males. Journal of Affective Disorders, 217, 60-65. doi:10.1016/j.jad.2017.03.072

Rivas, C., de Dios, J., Benac, M., \& Colomer, J. (2017). Análisis de los factores ligados al diagnóstico del trastorno por déficit de atención e hiperactividad en la infancia. Neurología, 32(7), 431-439. 
doi.org/10.1016/j.nrl.2016.01.006.

Rodríguez-Martos, A., \& Rosón, B. (2008). Definición y terminología. En Ministerio de Sanidad y Consumo (Ed.). Prevención de los problemas derivados del alcohol: $1^{a}$ Conferencia de prevención y promoción de la salud en la práctica clínica en España (pp. 39-48). Madrid, España: Ministerio de Sanidad y Consumo.

Rubiales, J. (2014). Perfil ejecutivo en niños con trastorno por déficit de atención con hiperactividad. Revista Iberoamericana de Diagnóstico y Evaluación - e Avaliação Psicológica, 38(2), 31-54.

van Emmerik-van Oortmerssen, K., van de Glind, G., van den Brink, W., Smit, F., Crunelle, C. L., Swets, M., \& Schoevers, R. A. (2012). Prevalence of attention-deficit hyperactivity disorder in substance use disorder patients: A meta-analysis and meta-regression analysis. Drug and Alcohol Dependence, 122(1-2), 1119. doi.org/10.1016/j.drugalcdep.2011.12.007-

Vázquez, M. J., Isorna, M., \& Redondo, L. (2020). Consumo de cannabis: $\measuredangle$ Es necesario un abordaje desde la perspectiva de género? En Nuevas aportaciones sobre el consumo de cannabis desde las ciencias sociales y de la salud (pp. 191-196). Andavira.

Willcutt, E. (2012). The prevalence of DSM-IV attention-deficit/hyperactivity disorder: A meta-analytic review. Neurotherapeutics, 9(3), 490-499. https://doi.org/10.1007/s13311-012-0135-8.

Winters, K. C., Stinchfield, R., Henly, G. A., \& Schwartz, R. (1990). Validity of adolescent self-report of alcohol and other drug involvement. International Journal of the Addictions, 25, 1379-1395.

doi.org/10.3109/10826089009068469. 